\title{
Cubic fourfolds containing a plane and a quintic del Pezzo surface
}

\author{
Asher Auel, Marcello Bernardara, Michele Bolognesi and Anthony \\ Várilly-Alvarado
}

\begin{abstract}
We isolate a class of smooth rational cubic fourfolds $X$ containing a plane whose associated quadric surface bundle does not have a rational section. This is equivalent to the nontriviality of the Brauer class $\beta$ of the even Clifford algebra over the K3 surface $S$ of degree 2 arising from $X$. Specifically, we show that in the moduli space of cubic fourfolds, the intersection of divisors $\mathcal{C}_{8} \cap \mathcal{C}_{14}$ has five irreducible components. In the component corresponding to the existence of a tangent conic, we prove that the general member is both pfaffian and has $\beta$ nontrivial. Such cubic fourfolds provide twisted derived equivalences between K3 surfaces of degrees 2 and 14, hence further corroboration of Kuznetsov's derived categorical conjecture on the rationality of cubic fourfolds.
\end{abstract}

\section{Introduction}

Let $X$ be a cubic fourfold, that is, a smooth cubic hypersurface $X \subset \mathbb{P}^{5}$ over the complex numbers. Determining the rationality of $X$ is an open problem in algebraic geometry. Some classes of rational cubic fourfolds have been described by Fano [Fan43], Tregub [Tre84, Tre93], and Beauville-Donagi [BD85]. In particular, pfaffian cubic fourfolds, defined by pfaffians of skew-symmetric $6 \times 6$ matrices of linear forms in $\mathbb{P}^{5}$, are rational; see [BD85, Prop. 5(ii)]. A cubic fourfold is pfaffian if and only if it contains a quintic del Pezzo surface; see [Bea00, Prop. 9.2 a)].

Hassett [Has00] describes, via lattice theory, Noether-Lefschetz divisors $\mathcal{C}_{d}$ in the moduli space $\mathcal{C}$ of cubic fourfolds, defined by the existence of 2-cycles not equivalent to a 2-dimensional linear section. For example, $\mathcal{C}_{14}$ is the closure of the locus of pfaffian cubic fourfolds and $\mathcal{C}_{8}$ is the locus of cubic fourfolds containing a plane. Certain of the divisors $\mathcal{C}_{d}$ consist of cubic fourfolds $X$ whose nonspecial cohomology is isomorphic to a Tate twist of the primitive middle cohomology of a polarized K3 surface $S$ of degree $d$; see [Has00, Thm. 1.0.2]. Such a K3 surface $S$ is said

Received 14 March 2013, accepted in final form 10 October 2013.

2010 Mathematics Subject Classification 11E20, 11E88, 14C30, 14F05, 14E08, 14F22, 14J28, 15A66

Keywords: cubic fourfold, quadric surface bundle, K3 surface, rationality, derived category

This journal is (C) Foundation Compositio Mathematica 2014. This article is distributed with Open Access under the terms of the Creative Commons Attribution Non-Commercial License, which permits non-commercial reuse, distribution, and reproduction in any medium, provided that the original work is properly cited. For commercial re-use, please contact the Foundation Compositio Mathematica.

Much of this work has been developed during visits of the authors at the Max Planck Institut für Mathematik in Bonn, Universität Duisburg-Essen, Université Rennes 1, ETH Zürich, and Rice University. The hospitality of each institute is warmly acknowledged. The first author was partially supported by NSF grant MSPRF DMS-0903039 and an NSA Young Investigator grant. The second author was partially supported by the SFB/TR 45 'Periods, moduli spaces, and arithmetic of algebraic varieties'. The fourth author was partially supported by NSF grant DMS-1103659. The authors would like to thank N. Addington, F. Charles, J.-L. Colliot-Thélène, B. Hassett, M.-A. Knus, R. Laza, E. Macrì, R. Parimala, P. Stellari, and V. Suresh for many helpful discussions. Comments from an anonymous referee, who is wholeheartedly thanked, enabled us to improve the focus and clarity of this text. 


\section{Auel, Bernardara, Bolognesi and VÁrilly-Alvarado}

to be associated with $X$. A natural suspicion, supported by Hassett's work [Has00, Has99], is that any rational cubic fourfold ought to have an associated K3 surface. For example, pfaffian cubic fourfolds have associated K3 surfaces of degree 14. Hassett [Has99, Thm. 4.2] identifies countably many divisors of $\mathcal{C}_{8}$ consisting of cubic fourfolds containing a plane whose Clifford invariant is trivial, implying rationality. Though lacking an associated K3 surface of degree 8, such cubic fourfolds do have associated K3 surfaces of other degrees. The work of Hassett and Tschinkel [HT01, §7] highlights the important role of effectivity of 2-cycles in such rationality considerations. While it is expected that the general cubic fourfold (and the general cubic fourfold containing a plane) is nonrational, at present not a single cubic fourfold is provably nonrational.

In this work, we study rational cubic fourfolds in $\mathcal{C}_{8} \cap \mathcal{C}_{14}$ with nontrivial Clifford invariant, hence not contained in the divisors of $\mathcal{C}_{8}$ described by Hassett. Let $A(X)$ be the lattice of algebraic 2-cycles on $X$ up to rational equivalence and let $d_{X}$ be the discriminant of the intersection form on $A(X)$. Our main result is a complete description of the irreducible components of $\mathcal{C}_{8} \cap \mathcal{C}_{14}$.

Theorem A. The intersection of Noether-Lefschetz divisors $\mathcal{C}_{8} \cap \mathcal{C}_{14}$ in the moduli space of cubic fourfolds has five irreducible components indexed by the discriminant $d_{X} \in\{21,29,32,36,37\}$ of a general member $X$. The Clifford invariant of a general cubic fourfold $X$ in $\mathcal{C}_{8} \cap \mathcal{C}_{14}$ is trivial if and only if $d_{X}$ is odd. Finally, the pfaffian locus is dense in the $d_{X}=32$ component.

In particular, the general cubic fourfold in the $d_{X}=32$ component of $\mathcal{C}_{8} \cap \mathcal{C}_{14}$ is rational and has nontrivial Clifford invariant; the existence of such a component substantiates an unproven assertion of Hassett [Has99, Rem. 4.3]. We also provide a geometric description of this component: its general member has a tangent conic to the sextic discriminant curve of the associated quadric surface bundle (see Proposition 6).

More recently, Kuznetsov [Kuz10] has established a semiorthogonal decomposition of the bounded derived category $\mathrm{D}^{\mathrm{b}}(X)=\left\langle\mathrm{A}_{X}, \mathscr{O}_{X}, \mathscr{O}_{X}(1), \mathscr{O}_{X}(2)\right\rangle$. The category $\mathrm{A}_{X}$ is essentially a noncommutative deformation of the derived category of a K3 surface. Based on evidence from known cases, as well as general categorical considerations, Kuznetsov conjectures that a cubic fourfold is rational if and only if there is an equivalence $\mathrm{A}_{X} \cong \mathrm{D}^{\mathrm{b}}(S)$ for some K3 surface $S$.

Shortly after the first version of this article appeared, Addington and Thomas [AT12] announced a groundbreaking result linking the Hodge-theoretic suspicion supported by Hassett's work and the derived categorical conjecture of Kuznetsov, at least for general cubic fourfolds.

If $X$ contains a plane, the projection from that plane makes $X$ birational to the total space of a quadric surface bundle $\widetilde{X} \rightarrow \mathbb{P}^{2}$ whose discriminant $D \subset \mathbb{P}^{2}$ is a sextic curve. Assuming that $D$ is smooth, the double cover $S \rightarrow \mathbb{P}^{2}$ branched along $D$ is a K3 surface of degree 2 and the even Clifford algebra gives a Brauer class $\beta \in \operatorname{Br}(S)$, called the Clifford invariant of $X$. In [Kuz10, Thm. 4.3], Kuznetsov establishes an equivalence $\mathrm{A}_{X} \cong \mathrm{D}^{\mathrm{b}}(S, \beta)$ with the bounded derived category of $\beta$-twisted sheaves on $S$.

By classical results in the theory of quadratic forms (see [KPS86, Thm. 6.3]), $\beta$ is trivial if and only if the quadric surface bundle $\widetilde{X} \rightarrow \mathbb{P}^{2}$ has a rational section (see also [Has99]), in which case $X$ is rational. On the divisors of $\mathcal{C}_{8}$ described by Hassett, $\beta \in \operatorname{Br}(S)$ is trivial and Kuznetsov's conjecture is verified. However, there is no example in the literature with $\beta$ nontrivial and for which there nevertheless exist a different $\mathrm{K} 3$ surface $S^{\prime}$ and an equivalence $\mathrm{D}^{\mathrm{b}}(S, \beta) \cong \mathrm{D}^{\mathrm{b}}\left(S^{\prime}\right)$ explaining the rationality of $X$. One application of our results is the existence of such examples.

Corollary B. Let $X$ be a general member of the $d_{X}=32$ component of $\mathcal{C}_{8} \cap \mathcal{C}_{14}$. Then there exist a $K 3$ surface $S^{\prime}$ of degree 14 and a nontrivial twisted derived equivalence $\mathrm{D}^{\mathrm{b}}(S, \beta) \cong \mathrm{D}^{\mathrm{b}}\left(S^{\prime}\right)$. 


\section{Cubic fourfolds Containing a Plane and a quintic Del Pezzo surface}

The existence of such cubic fourfolds is not a priori clear. First, while the locus of pfaffian cubic fourfolds is dense in $\mathcal{C}_{14}$, it is not true that the locus of pfaffians containing a plane is dense in (all components of) $\mathcal{C}_{8} \cap \mathcal{C}_{14}$. As for the general study of quadric bundles over surfaces, there do exist conic bundles over surfaces, without rational sections, whose total space is smooth projective rational: these are classified over rational minimal surfaces [Sho84] (see also [BB13]); over $\mathbb{P}^{2}$ their discriminant curves have degree at most five [Bea77]. However, there is no analogous classification of quadric surface bundles over surfaces with smooth projective rational total space and without rational sections. Indeed, there do not even exist any explicit examples in the literature. Our results provide such examples arising from cubic fourfolds containing a plane.

The structure of this paper is as follows. In $\S 1$, we study Hodge-theoretic and geometric conditions for the nontriviality of the Clifford invariant (see Proposition 2 and Corollary 3). In $\S 2$, we analyze the irreducible components of $\mathcal{C}_{8} \cap \mathcal{C}_{14}$, proving the first two statements of Theorem A. Throughout, we use the work of Looijenga [Loo09] and Laza [Laz10], as adapted by Mayanskiy [May11], on the realizability of lattices of algebraic cycles on a cubic fourfold. In $\S 3$, we recall some elements of the theory of homological projective duality and prove Corollary B. Finally, in $\S 4$, we prove the final statement of Theorem A, that the pfaffian locus is dense in the $d_{X}=32$ component of $\mathcal{C}_{8} \cap \mathcal{C}_{14}$, by expliciting a single point in the intersection. For the verification, we are aided by Magma [BCP93], adapting some of the computational techniques developed in [HVV11].

\section{Nontriviality criteria for Clifford invariants}

In this section, by means of straightforward lattice-theoretic calculations, we describe a class of cubic fourfolds containing a plane with nontrivial Clifford invariant.

If $(H, b)$ is a $\mathbb{Z}$-lattice and $A \subset H$ is a sublattice, then the orthogonal complement $A^{\perp}=\{v \in H: b(v, A)=0\}$ is a saturated sublattice (that is, $\left.A^{\perp}=A^{\perp} \otimes_{\mathbb{Z}} \mathbb{Q} \cap H\right)$ and is thus a primitive sublattice (in other words, $H / A^{\perp}$ is torsion free). Denote by $d(H, b) \in \mathbb{Z}$ the discriminant, that is, the determinant of a Gram matrix.

Let $X$ be a smooth cubic fourfold over $\mathbb{C}$. The integral Hodge conjecture holds for $X$ (by [Voi07b, Thm. 18], following [Mur77] and [Zuc77]) and we denote by $A(X)=H^{4}(X, \mathbb{Z}) \cap H^{2,2}(X)$ the lattice of integral middle Hodge classes; it coincides with the Chow group of codimension 2 cycles up to rational equivalence.

Now suppose that $X$ contains a plane $P$ and let $\pi: \widetilde{X} \rightarrow \mathbb{P}^{2}$ be the quadric surface bundle defined by blowing up and projecting away from $P$. Let $\mathscr{C}_{0}$ be the even Clifford algebra of $\pi$; see [Kuz08] or $[\mathrm{ABB} 13, \S 1.5]$. We call the plane $P$ good if $\pi$ has simple degeneration, that is, the fibers of $\pi$ have at most isolated singularities. This is equivalent to $X$ not containing another plane intersecting $P$; see [Voi86, $\S 1$ Lemme 2]. This is also equivalent to the smoothness of the discriminant divisor $D \subset \mathbb{P}^{2}$ (see [ABB13, Prop. 1.2.5]), which is sextic curve. In this case, the discriminant cover $f: S \rightarrow \mathbb{P}^{2}$ branched along $D$ is a smooth K3 surface of degree 2, and $\mathscr{C}_{0}$ defines an Azumaya quaternion algebra over $S$; see [Kuz08, Prop. 3.13]. We refer to the Brauer class $\beta \in \operatorname{Br}(S)[2]$ of $\mathscr{C}_{0}$ as the Clifford invariant of $X$.

Let $h \in H^{2}(X, \mathbb{Z})$ be the hyperplane class defined by the embedding $X \subset \mathbb{P}^{5}$. The transcendental lattice $T(X)$, the nonspecial cohomology lattice $K$, and the primitive cohomology lattice $H^{4}(X, \mathbb{Z})_{0}$ are the orthogonal complements (with respect to the cup product polarization $\left.b_{X}\right)$ of $A(X),\left\langle h^{2}, P\right\rangle$, and $\left\langle h^{2}\right\rangle$ inside $H^{4}(X, \mathbb{Z})$, respectively. Thus $T(X) \subset K \subset H^{4}(X, \mathbb{Z})_{0}$, with $T(X)=K$ for a very general cubic fourfold containing a plane; see the proof of [Voi86, 


\section{Auel, Bernardara, Bolognesi and VÁrilly-Alvarado}

$\S 1$ Prop. 2]. There are polarized Hodge structures on $T(X), K$, and $H^{4}(X, \mathbb{Z})_{0}$ by restricting from $H^{4}(X, \mathbb{Z})$.

Similarly, let $S$ be a smooth integral projective surface over $\mathbb{C}$ and let $\operatorname{NS}(S)=H^{2}(S, \mathbb{Z}) \cap$ $H^{1,1}(S)$ be its Néron-Severi lattice. Let $h_{1} \in \mathrm{NS}(S)$ be a fixed anisotropic class. The transcendental lattice $T(S)$ and the primitive cohomology $H^{2}(S, \mathbb{Z})_{0}$ are the orthogonal complements (with respect to the cup product polarization $\left.b_{S}\right)$ of $\mathrm{NS}(S)$ and $\left\langle h_{1}\right\rangle$ inside $H^{2}(S, \mathbb{Z})$, respectively. If $f: S \rightarrow \mathbb{P}^{2}$ is a double cover, then we take $h_{1}$ to be the class of $f^{*} \mathscr{O}_{\mathbb{P}^{2}}(1)$.

Let $F(X)$ be the Fano variety of lines in $X$ and let $W \subset F(X)$ be the divisor consisting of the lines meeting $P$. Then $W$ is identified with the relative Hilbert scheme of lines in the fibers of $\pi$. Its Stein factorization $W \stackrel{p}{\rightarrow} S \stackrel{f}{\rightarrow} \mathbb{P}^{2}$ displays $W$ as a smooth conic bundle over the discriminant cover. The Abel-Jacobi map

$$
\Phi: H^{4}(X, \mathbb{Z}) \rightarrow H^{2}(W, \mathbb{Z})
$$

is an isomorphism of $\mathbb{Q}$-Hodge structures $\Phi: H^{4}(X, \mathbb{Q}) \rightarrow H^{2}(W, \mathbb{Q})(-1)$; see [Voi86, $\S 1$ Prop. 1]. Finally, there is an injective (see [Voi07a, Lemma 7.28]) morphism $p^{*}: H^{2}(S, \mathbb{Z}) \rightarrow H^{2}(W, \mathbb{Z})$ of polarized Hodge structures. Voisin [Voi86, $\S 1$ Prop. 2] proves that $\Phi(K) \subset p^{*} H^{2}(S, \mathbb{Z})_{0}(-1)$ is a polarized Hodge substructure of index 2. Here, the Tate twist $(-1)$ increases the weight by 2 and changes the sign of the bilinear form. We have the following amplification.

Proposition 1. Let $X$ be a smooth cubic fourfold containing a good plane. Then we have that $\Phi(T(X)) \subset p^{*} T(S)(-1)$ is a polarized Hodge substructure of index $\epsilon$ dividing 2. In particular, rk $A(X)=\operatorname{rkNS}(S)+1$ and $d(A(X))=2^{2(\epsilon-1)} d(\mathrm{NS}(S))$.

Proof. Using [Voi86, $\S 1$ Lemme 3] it is not hard to check that $\Phi(T(X)) \subset p^{*} T(S)(-1)$; it remains to compute the index of this inclusion. Since $T(X) \subset K$ and $T(S)(-1) \subset H^{2}(S, \mathbb{Z})_{0}(-1)$ are saturated (hence primitive) sublattices, an application of the snake lemma shows that

$$
p^{*} T(S)(-1) / \Phi(T(X)) \subset p^{*} H^{2}(S, \mathbb{Z})_{0} / \Phi(K) \cong \mathbb{Z} / 2 \mathbb{Z},
$$

hence the index of $\Phi(T(X))$ in $p^{*} T(S)(-1)$ divides 2 .

We now verify the final claims. We have $\operatorname{rk} K=\operatorname{rk} H^{2}(X, \mathbb{Z})-2=\operatorname{rk} T(X)+\operatorname{rk} A(X)-2$ and $\operatorname{rk} H^{2}(S, \mathbb{Z})_{0}=\operatorname{rk} H^{2}(S, \mathbb{Z})-1=\operatorname{rk} T(S)+\operatorname{rkNS}(S)-1$ (since $P, h^{2}$, and $h_{1}$ are anisotropic vectors), while $\operatorname{rk} K=\operatorname{rk} H^{2}(S, \mathbb{Z})_{0}$ and $\operatorname{rk} T(X)=\operatorname{rk} T(S)$ by [Voi86, $\S 1$ Prop. 2] and the above, respectively. The discriminant calculation follows from standard lattice theory.

If $S$ has Picard rank 1, then $X$ has no associated K3 surface in the sense of Hassett. Thus, if we are looking for rational cubic fourfolds, we must consider $S$ with Picard rank at least 2 . Let $Q \in A(X)$ be the class of a fiber of $\pi: \widetilde{X} \rightarrow \mathbb{P}^{2}$. Then $P+Q=h^{2}$; see [Voi86, $\S 1$ ].

Proposition 2. Let $X$ be a smooth cubic fourfold containing a good plane $P$. If $A(X)$ has rank 3 and even discriminant (for example, if the K3 surface $S$ of degree 2 has Picard rank 2 and even Néron-Severi discriminant) then the Clifford invariant $\beta \in \operatorname{Br}(S)$ of $X$ is nontrivial.

Proof. The Clifford invariant $\beta \in \operatorname{Br}(S)$ of the quadric surface bundle $\pi: \widetilde{X} \rightarrow \mathbb{P}^{2}$ is trivial if and only if $\pi$ has a rational section; see [KPS86, Thm. 6.3] or [Sch85, 2 Thm. 14.1 and Lemma 14.2]. Such a section exists if and only if there exists an algebraic cycle $R \in A(X)$ such that $R . Q=1$; see [Has99, Thm. 3.1] or [Kuz10, Prop. 4.7].

Suppose that such a cycle $R$ exists and consider the sublattice $\left\langle h^{2}, Q, R\right\rangle \subset A(X)$. It is straightforward to see that its intersection form has a Gram matrix whose determinant is congruent to 5 modulo 8 for any possible choice of $R$ (see [Has99, Lemma 4.4]), so this lattice cannot 


\section{Cubic fourfolds containing a Plane and a quintic del Pezzo surface}

be a finite index sublattice of $A(X)$, which has even discriminant by hypothesis. Hence no such 2 -cycle $R$ exists and thus $\beta$ is nontrivial. The claim follows from Proposition 1.

We now provide an explicit geometric condition for the nontriviality of the Clifford invariant, which will be necessary in $\S 4$. We say that a cubic fourfold $X$ containing a plane has a tangent conic if there exists a conic $C \subset \mathbb{P}^{2}$ everywhere tangent to the discriminant curve $D \subset \mathbb{P}^{2}$ of the associated quadric surface bundle.

Corollary 3. Let $X$ be a smooth cubic fourfold containing a good plane. If $X$ has a tangent conic and the K3 surface $S$ has Picard rank 2, then the Clifford invariant $\beta \in \operatorname{Br}(S)$ of $X$ is nontrivial.

Proof. Consider the pull back of the cycle class of $C$ to $S$ via the discriminant double cover $f: S \rightarrow \mathbb{P}^{2}$. Then $f^{*} C$ has two components $C_{1}$ and $C_{2}$. The sublattice of $\mathrm{NS}(S)$ generated by $h_{1}=f^{*} \mathscr{O}_{\mathbb{P}^{2}}(1)=\left(C_{1}+C_{2}\right) / 2$ and $C_{1}$ has discriminant -8. As $S$ has Picard rank 2, the classes $h_{1}$ and $C_{1}$ generate $\mathrm{NS}(S)$ (see [EJ08, $\S 2$ ] for further details). Now apply Proposition 2.

\section{The Clifford invariant on $\mathcal{C}_{8} \cap \mathcal{C}_{14}$}

In this section, we first prove that $\mathcal{C}_{8} \cap \mathcal{C}_{14}$ has five irreducible components and we describe each of them in lattice-theoretic terms. We then completely analyze the (non)triviality of the Clifford invariant of the general cubic fourfold (that is, such that $A(X)$ has rank 3 ) in each irreducible component. One of the components corresponds to cubic fourfolds containing a plane and having a tangent conic (that is, those considered in Corollary 3), where we already know the nontriviality of the Clifford invariant. Another component corresponds to cubic fourfolds containing two disjoint planes, where we already know the triviality of the Clifford invariant. There are another two components of $\mathcal{C}_{8} \cap \mathcal{C}_{14}$ whose general elements have trivial Clifford invariant (see Proposition 5).

A cubic fourfold $X$ is in $\mathcal{C}_{8}$ or $\mathcal{C}_{14}$ if and only if $A(X)$ has a primitive sublattice $K_{8}=\left\langle h^{2}, P\right\rangle$ or $K_{14}=\left\langle h^{2}, T\right\rangle$. This follows from the definition of $\mathcal{C}_{d}$ and because for any $d \not \equiv 0 \bmod 9$ there is a unique lattice (up to isomorphism) of rank 2 that represents 3 and has discriminant $d$.

Thus a cubic fourfold $X$ in $\mathcal{C}_{8} \cap \mathcal{C}_{14}$ has a sublattice $\left\langle h^{2}, P, T\right\rangle \subset A(X)$ with Gram matrix

$\begin{array}{cccc} & h^{2} & P & T \\ h^{2} & 3 & 1 & 4 \\ P & 1 & 3 & \tau \\ T & 4 & \tau & 10\end{array}$

for some $\tau \in \mathbb{Z}$ depending on $X$. There may be a priori restrictions on the possible values of $\tau$.

Denote by $A_{\tau}$ the lattice of rank 3 whose bilinear form has Gram matrix (1). We will write $\mathcal{C}_{\tau}=\mathcal{C}_{A_{\tau}} \subset \mathcal{C}$ for the locus of smooth cubic fourfolds such that there is a primitive embedding $A_{\tau} \subset A(X)$ of lattices preserving $h^{2}$. If nonempty, each $\mathcal{C}_{\tau}$ is a subvariety of codimension 2 by a variant of the proof of [Has00, Thm. 3.1.2].

We will use the work of Laza [Laz10], Looijenga [Loo09], and Mayanskiy [May11, Thm. 6.1] to classify exactly which values of $\tau$ are supported by cubic fourfolds. This proves the first assertion in Theorem A.

Theorem 4. The irreducible components of $\mathcal{C}_{8} \cap \mathcal{C}_{14}$ are the subvarieties $\mathcal{C}_{\tau}$ for $\tau \in\{-1,0,1,2,3\}$. Moreover, the general cubic fourfold $X$ in $\mathcal{C}_{\tau}$ satisfies $A(X) \cong A_{\tau}$. 


\section{Auel, Bernardara, Bolognesi and Várilly-Alvarado}

Proof. By construction, $\mathcal{C}_{8} \cap \mathcal{C}_{14}$ is the union of $\mathcal{C}_{\tau}$ for all $\tau \in \mathbb{Z}$. First, we determine the values of $\tau$ for which the component $\mathcal{C}_{\tau}$ is possibly nonempty. If $X$ is a smooth cubic fourfold, then $A(X)$ is positive definite by the Riemann bilinear relations. Hence, to be realized as a sublattice of some $A(X)$, the lattice $A_{\tau}$ must be positive definite, which by Sylvester's criterion is equivalent to $A_{\tau}$ having positive discriminant. As $d\left(A_{\tau}\right)=-3 \tau^{2}+8 \tau+32$, the only values of $\tau$ making a positive discriminant are $-2,-1,0,1,2,3,4$.

Then, we prove that $\mathcal{C}_{\tau}$ is empty for $\tau=-2,4$ by demonstrating roots (that is, primitive vectors of norm 2) in $A_{\tau, 0}=\left\langle h^{2}\right\rangle^{\perp}$ (see [Voi86, §4 Prop. 1], [Loo09, § 2], or [Laz10, Def. 2.16] for details on roots). Indeed, the vectors $(1,-3,0)$ and $(0,-4,1)$ form a basis for $A_{\tau, 0} \subset A_{\tau}$; for $\tau=-2$, we find short roots $(-2,2,1)$ and $(2,-10,1)$; for $\tau=4$, we find short roots $\pm(1,1,-1)$. Hence $\mathcal{C}_{\tau}$ is possibly nonempty only for $\tau \in\{-1,0,1,2,3\}$. The corresponding discriminants $d\left(A_{\tau}\right)$ are $21,32,37,36,29$.

For the remaining values of $\tau$, we prove that $\mathcal{C}_{\tau}$ is nonempty. To this end, we verify conditions 1)-6) of [May11, Thm. 6.1], proving that $A_{\tau}=A(X)$ for some cubic fourfold $X$. Condition 1) is true by definition. For condition 2$)$, letting $v=(x,-3 x-4 y, y) \in A_{\tau, 0}$, we see that

$$
b(v, v)=2\left(12 x^{2}+(36-3 \tau) x y+(29-4 \tau) y^{2}\right)
$$

is even. For condition 5$)$, letting $w=(x, y, z) \in A_{\tau}$, we compute that

$$
b\left(h^{2}, w\right)^{2}-b(w, w)=2\left(3 x^{2}-y^{2}+z^{2}+2 x y+8 x z+(4-\tau) y z\right)
$$

is even. For conditions 3)-4), given each of the five values of $\tau$, we use standard Diophantine techniques to prove the nonexistence of short and long roots of (2).

Finally, for condition 6 ), let $q_{K_{\tau}}: A_{\tau}^{*} / A_{\tau} \rightarrow \mathbb{Q} / 2 \mathbb{Z}$ be the discriminant form of (3), restricted to the discriminant group $A_{\tau}^{*} / A_{\tau}$ of the lattice $A_{\tau}$. Appealing to Nikulin [Nik79, Cor. 1.10.2], it suffices to check that the signature satisfies $\operatorname{sgn}\left(q_{K_{\tau}}\right) \equiv 0 \bmod 8$; see [May11, Rem. 6.3]. Employing the notation of [Nik79, Prop. 1.8.1], we compute the finite quadratic form $q_{K_{\tau}}$ in each case:

\begin{tabular}{|c||c|c|c|c|c|}
\hline$\tau$ & -1 & 0 & 1 & 2 & 3 \\
\hline$d\left(A_{\tau}\right)$ & 21 & 32 & 37 & 36 & 29 \\
\hline$A_{\tau}^{*} / A_{\tau}$ & $\mathbb{Z} / 3 \mathbb{Z} \times \mathbb{Z} / 7 \mathbb{Z}$ & $\mathbb{Z} / 2 \mathbb{Z} \times \mathbb{Z} / 16 \mathbb{Z}$ & $\mathbb{Z} / 37 \mathbb{Z}$ & $\mathbb{Z} / 2 \mathbb{Z} \times \mathbb{Z} / 2 \mathbb{Z} \times \mathbb{Z} / 9 \mathbb{Z}$ & $\mathbb{Z} / 29 \mathbb{Z}$ \\
\hline$q_{K_{\tau}}$ & $q_{1}^{3}(3) \oplus q_{1}^{7}(7)$ & $q_{3}^{2}(2) \oplus q_{1}^{2}\left(2^{4}\right)$ & $q_{\theta}^{37}(37)$ & $q_{3}^{2}(2) \oplus q_{1}^{2}(2) \oplus q_{1}^{3}\left(3^{2}\right)$ & $q_{\theta}^{29}(29)$ \\
\hline
\end{tabular}

where $\theta$ represents a nonsquare class modulo the respective odd prime. In each case of (4), we verify the signature condition using the formulas in [Nik79, Prop. 1.11.2].

Finally, for the five values of $\tau$, we prove that $\mathcal{C}_{\tau}$ is irreducible. As the rank of $A(X)$ is an upper-semicontinuous function on $\mathcal{C}$, the general cubic fourfold $X$ in $\mathcal{C}_{8} \cap \mathcal{C}_{14}$ has $A(X)$ of rank 3 (by the argument above), of which $A_{\tau}$ is a finite index sublattice for some $\tau$. Each proper finite overlattice $B$ of $A_{\tau}$ such that $B$ (along with its sublattices $K_{8}$ and $K_{14}$ ) is primitively embedded into $H^{4}(X, \mathbb{Z})$ will give rise to an irreducible component of $\mathcal{C}_{\tau}$. We will prove that no such proper finite overlattices exist. For $\tau \in\{21,37,29\}$, the discriminant of $A_{\tau}$ is squarefree, so there are no proper finite overlattices. In the case $\tau=0,2$, we note that $B_{0}=\left\langle h^{2}\right\rangle^{\perp}$ is a proper finite overlattice of the binary lattice $A_{\tau, 0}$ (as $\left\langle h^{2}\right\rangle \subset B$ is assumed primitive). We then directly compute that each such $B_{0}$ has long roots (that is, vectors of norm 6 whose pairing with any other vector is divisible by 3 ). Therefore, no such proper finite overlattices exist.

We now address the question of the (non)triviality of the Clifford invariant. This proves the 


\section{Cubic fourfolds containing a Plane and a quintic del Pezzo surface}

second assertion of Theorem A.

Proposition 5. Let $X$ be a general cubic fourfold in $\mathcal{C}_{8} \cap \mathcal{C}_{14}$ (so that $A(X)$ has rank 3). The Clifford invariant $\beta \in \operatorname{Br}(S)$ of $X$ is trivial if and only if $\tau$ is odd.

Proof. If $\tau$ is odd then, as in the proof of Proposition 6, $(P+T) \cdot Q=-\tau$ is odd, hence the Clifford invariant $\beta \in \operatorname{Br}(S)$ is trivial by an application of the criteria in [Has99, Thm. 3.1] or [Kuz10, Prop. 4.7] (see the proof of Proposition 2). If $\tau$ is even, then $A_{d}=A(X)$ has rank 3 and even discriminant, hence $\beta$ is nontrivial by Proposition 2 .

For $\tau=-1$, the component $\mathcal{C}_{\tau}$ consists of cubic fourfolds containing two disjoint planes; see [Has00, 4.1.3]. We now give a geometric description of the general member of the component $\mathcal{C}_{\tau}$ for $\tau=0$ (that is, where $d_{X}=32$ ).

Proposition 6. Let $X$ be a smooth cubic fourfold containing a good plane $P$ and having a tangent conic such that $A(X)$ has rank 3. Then $X$ is in the component $\mathcal{C}_{\tau}$ for $\tau=0$.

Proof. Since $X$ has a tangent conic and $A(X)$ has rank $3, A(X)$ has discriminant 8 or 32 and $X$ has nontrivial Clifford invariant by Proposition 1 and Corollary 3 . As the sublattice $\left\langle h^{2}, P\right\rangle \subset A(X)$ is primitive, we can choose a class $T \in A(X)$ such that $\left\langle h^{2}, P, T\right\rangle \subset A(X)$ has discriminant 32. Adjusting $T$ by a multiple of $P$, we can assume that $h^{2} . T=4$. Write $\tau=P . T$.

Adjusting $T$ by multiples of $h^{2}-3 P$ keeps $h^{2} \cdot T=4$ and adjusts $\tau$ by multiples of 8 . A similar trick is employed in [AT12, Prop. 4.2]. The discriminant being 32, we are left with two possible choices $(\tau=0,4)$ for the Gram matrix of $\left\langle h^{2}, P, T\right\rangle$ up to isomorphism:

$\begin{array}{cccccccc} & h^{2} & P & T & & h^{2} & P & T \\ h^{2} & 3 & 1 & 4 & h^{2} & 3 & 1 & 4 \\ P & 1 & 3 & 0 & P & 1 & 3 & 4 \\ T & 4 & 0 & 10 & T & 4 & 4 & 12\end{array}$

In these cases, we compute that $K \cap\left\langle h^{2}, P, T\right\rangle$ (that is, the orthogonal complement of $\left\langle h^{2}, P\right\rangle$ in $\left.\left\langle h^{2}, P, T\right\rangle\right)$ is generated by $3 h^{2}-P-2 T$ and $h^{2}+P-T$ and has discriminant 16 and 5 , respectively. We calculate that $\mathrm{NS}(S) \cap H^{2}(S, \mathbb{Z})_{0}$ (that is, the orthogonal complement of $\left\langle h_{1}\right\rangle$ in $\mathrm{NS}(S)$ ) is generated by $h_{1}-C_{1}$ and has discriminant -4 (see Corollary 3 for definitions). Arguing as in the proof of Proposition 1, there is a lattice inclusion $\Phi\left(K \cap\left\langle h^{2}, P, T\right\rangle\right) \subset \mathrm{NS}(S) \cap H^{2}(S, \mathbb{Z})_{0}(-1)$ having index dividing 2 , which rules out the second case above by comparing discriminants.

In Proposition 5, we isolate three classes of smooth cubic fourfolds $X \in \mathcal{C}_{8} \cap \mathcal{C}_{14}$ with trivial Clifford invariant. While the component $\mathcal{C}_{\tau}$ for $\tau=-1$ is in the complement of the pfaffian locus (see [Tre93, Prop. 1b]), we wonder if the pfaffian locus is dense in the other four components.

\section{The twisted derived equivalence}

Homological projective duality (HPD) can be used to obtain a significant semiorthogonal decomposition of the derived category of a pfaffian cubic fourfold. As the universal pfaffian variety is singular, a noncommutative resolution of singularities is required to establish HPD in this case. A noncommutative resolution of singularities of a scheme $Y$ is a coherent $\mathscr{O}_{Y \text {-algebra } \mathscr{R}}$ with finite homological dimension that is generically a matrix algebra (these properties translate to "smoothness" and "birational to $Y$ " from the categorical language). We refer to [Kuz07] for details on HPD. The following is a straightforward application of HPD for Grassmannians ([Kuz06]). 


\section{Auel, Bernardara, Bolognesi and VÁrilly-Alvarado}

Theorem 7. Let $W$ be a $\mathbb{C}$-vector space of dimension 6 and let $Y \subset \mathbb{P}\left(\wedge^{2} W^{\vee}\right)$ be the universal pfaffian cubic hypersurface. There exists a noncommutative resolution of singularities $(Y, \mathscr{R})$ that is HP dual to the Grassmannian $\operatorname{Gr}(2, W)$. In particular, the bounded derived category of a smooth pfaffian cubic fourfold $X$ admits a semiorthogonal decomposition

$$
\mathrm{D}^{\mathrm{b}}(X)=\left\langle\mathrm{D}^{\mathrm{b}}\left(S^{\prime}\right), \mathscr{O}_{X}, \mathscr{O}_{X}(1), \mathscr{O}_{X}(2)\right\rangle
$$

where $S^{\prime}$ is a smooth K3 surface of degree 14. In particular, $\mathrm{A}_{X} \cong \mathrm{D}^{\mathrm{b}}\left(S^{\prime}\right)$.

Assuming Theorem A, we can now give a proof of Corollary B.

Proof of Corollary B. By Theorem A, $X$ is a smooth pfaffian cubic fourfold containing a good plane with nontrivial Clifford invariant $\beta \in \operatorname{Br}(S)$. Being pfaffian, $X$ is rational. Let $S^{\prime}$ be the K3 surface of degree 14 arising from Theorem 7 via projective duality. Then by [Kuz10, Thm. 4.3] and Theorem 7 , the category $\mathrm{A}_{X}$ is equivalent to both $\mathrm{D}^{\mathrm{b}}(S, \beta)$ and $\mathrm{D}^{\mathrm{b}}\left(S^{\prime}\right)$.

Remark 8. By [HS05, Rem. 7.10], given any K3 surface $S$ and any nontrivial $\beta \in \operatorname{Br}(S)$, there is no equivalence between $\mathrm{D}^{\mathrm{b}}(S, \beta)$ and $\mathrm{D}^{\mathrm{b}}(S)$. Thus any $X$ as in Corollary B validates Kuznetsov's conjecture, but not via the K3 surface $S$. Moreover, $S$ and $S^{\prime}$ are twisted Fourier-Mukai partners: by [CS07, Thm. 5.1], the equivalence $\mathrm{D}^{\mathrm{b}}(S, \beta) \cong \mathrm{D}^{\mathrm{b}}\left(S^{\prime}\right)$ is a Fourier-Mukai functor whose kernel is a $\beta^{-1} \otimes \mathscr{O}_{S^{\prime}}$-twisted complex on $S \times S^{\prime}$. Hence, by [HS05, Thm. 4.3], $S$ and $S^{\prime}$ have Hodge isogenous (twisted) transcendental lattices.

\section{A pfaffian containing a plane}

In this section, we prove the final claim of Theorem A by exhibiting a smooth pfaffian cubic fourfold $X$ containing a good plane, having a tangent conic, and such that $A(X)$ has rank 3 . Indeed, by Propositions 2 and 6, such an $X$ has nontrivial Clifford invariant and is in the $\tau=0$ (that is, $d_{X}=32$ ) component of $\mathcal{C}_{8} \cap \mathcal{C}_{14}$. In particular, the pfaffian locus nontrivially intersects, and hence is dense in (since it is open in $\mathcal{C}_{14}$ ), the component $\mathcal{C}_{\tau}$ with $\tau=0$.

Theorem 9. Let $A$ be the $6 \times 6$ antisymmetric matrix

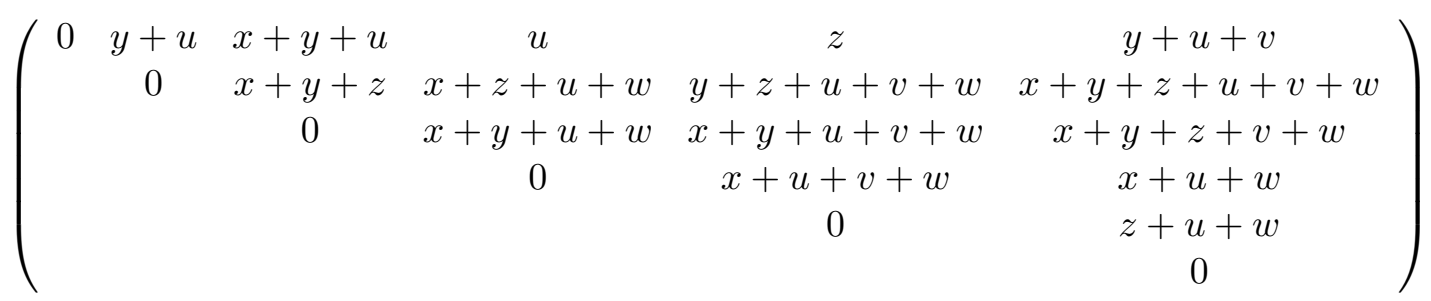

of linear forms in $\mathbb{Q}[x, y, z, u, v, w]$ and let $X \subset \mathbb{P}^{5}$ be the cubic fourfold defined by the vanishing of the pfaffian of $A$ :

$$
\begin{aligned}
& (x-4 y-z) u^{2}+(-x-3 y) u v+(x-3 y) u w+(x-2 y-z) v w-2 y v^{2}+x w^{2} \\
& +\left(2 x^{2}+x z-4 y^{2}+2 z^{2}\right) u+\left(x^{2}-x y-3 y^{2}+y z-z^{2}\right) v+\left(2 x^{2}+x y+3 x z-3 y^{2}+y z\right) w \\
& +x^{3}+x^{2} y+2 x^{2} z-x y^{2}+x z^{2}-y^{3}+y z^{2}-z^{3} .
\end{aligned}
$$

Then:

(i) $X$ is smooth, rational, and contains the plane $P=\{x=y=z=0\}$. 


\section{Cubic fourfolds Containing a Plane and a quintic Del Pezzo surface}

(ii) The discriminant divisor $D \subset \mathbb{P}^{2}$ of the quadric surface bundle $\pi: \tilde{X} \rightarrow \mathbb{P}^{2}$ is the sextic curve given by the vanishing of

$$
\begin{aligned}
d=x^{6} & +6 x^{5} y+12 x^{5} z+x^{4} y^{2}+22 x^{4} y z+28 x^{3} y^{3}-38 x^{3} y^{2} z+46 x^{3} y z^{2}+4 x^{3} z^{3} \\
& +24 x^{2} y^{4}-4 x^{2} y^{3} z-37 x^{2} y^{2} z^{2}-36 x^{2} y z^{3}-4 x^{2} z^{4}+48 x y^{4} z-24 x y^{3} z^{2} \\
& +34 x y^{2} z^{3}+4 x y z^{4}+20 y^{5} z+20 y^{4} z^{2}-8 y^{3} z^{3}-11 y^{2} z^{4}-4 y z^{5} .
\end{aligned}
$$

This curve is smooth; in particular, $\pi$ has simple degeneration and the discriminant cover is a smooth $K 3$ surface $S$ of degree 2.

(iii) The conic $C \subset \mathbb{P}^{2}$ defined by the vanishing of $x^{2}+y z$ is tangent to the degeneration divisor $D$ at six points (five of which are distinct).

(iv) The K3 surface $S$ has (geometric) Picard rank 2.

In particular, the Clifford invariant of $X$ is geometrically nontrivial.

Proof. An application of the jacobian criterion shows that $X$ and $D$ are smooth. The inclusion $P \subset X$ is checked by inspecting the expression for $\operatorname{pf}(A)$; every monomial is divisible by $x, y$ or $z$. Rationality comes from being a pfaffian cubic fourfold. Smoothness of $D$ and $X$ implies that $\pi$ has simple degeneration; see [HVV11, Rem. 7.1] or [ABB13, Prop. 1.6]. This proves parts (i) and (ii).

For part (iii), we write the equation for the degeneration divisor as $d=\left(x^{2}+y z\right) f+g^{2}$, where

$$
\begin{aligned}
f= & x^{4}+6 x^{3} y+12 x^{3} z+x^{2} y^{2}+21 x^{2} y z-25 x^{2} z^{2}+28 x y^{3} \\
& \quad-24 x y^{2} z+34 x y z^{2}+4 x z^{3}+20 y^{4}-5 y^{3} z-8 y^{2} z^{2}-11 y z^{3}-4 z^{4}, \\
g= & 2 x y^{2}+5 y^{2} z-5 x^{2} z .
\end{aligned}
$$

Hence the conic $C \subset \mathbb{P}^{2}$ defined by $x^{2}+y z$ is tangent to $D$ along the zero-dimensional scheme of length 6 given by the intersection of $C$ and the vanishing locus of $g$.

For part (iv), the surface $S$ is the smooth sextic in $\mathbb{P}(1,1,1,3)=\operatorname{Proj} \mathbb{Q}[x, y, z, w]$ given by $w^{2}=d(x, y, z)$, which is the double cover of $\mathbb{P}^{2}$ branched along $D$. In these coordinates, the discriminant cover $f: S \rightarrow \mathbb{P}^{2}$ is simply the restriction to $S$ of the projection $\mathbb{P}(1,1,1,3)-\rightarrow \mathbb{P}^{2}$ away from the hyperplane $\{w=0\}$. Let $C \subset \mathbb{P}^{2}$ be the conic from part (iii). As discussed in Corollary 3 , the curve $f^{*} C$ consists of two (-2)-curves $C_{1}$ and $C_{2}$. These curves generate a sublattice of $\operatorname{NS}(S)$ of rank 2 . Hence $\rho(\bar{S}) \geqslant \rho(S) \geqslant 2$, where $\bar{S}=S \times_{\mathbb{Q}} \mathbb{C}$.

We show next that $\rho(\bar{S}) \leqslant 2$. Write $S_{p}$ for the reduction $\bmod p$ of $S$ and $\bar{S}_{p}=S_{p} \times_{\mathbb{F}_{p}} \overline{\mathbb{F}}_{p}$. Let $\ell \neq 3$ be a prime and write $\phi(t)$ for the characteristic polynomial of the action of the absolute Frobenius on $H_{\text {ét }}^{2}\left(\bar{S}_{3}, \mathbb{Q}_{\ell}\right)$. Then $\rho\left(\bar{S}_{3}\right)$ is bounded from above by the number of roots of $\phi(t)$ that are of the form $3 \zeta$, where $\zeta$ is a root of unity [vLui07, Prop. 2.3]. Combining the Lefschetz trace formula with Newton's identities and the functional equation that $\phi(t)$ satisfies, it is possible to calculate $\phi(t)$ from the knowledge of $\# S\left(\mathbb{F}_{3^{n}}\right)$ for $1 \leqslant n \leqslant 11$; see [vLui07] for details.

Let $\widetilde{\phi}(t)=3^{-22} \phi(3 t)$, so that the number of roots of $\widetilde{\phi}(t)$ that are roots of unity gives an upper bound for $\rho\left(\bar{S}_{3}\right)$. Using Magma, we compute

$\widetilde{\phi}(t)=\frac{1}{3}(t-1)^{2}\left(3 t^{20}+t^{19}+t^{17}+t^{16}+2 t^{15}+3 t^{14}+t^{12}+3 t^{11}+2 t^{10}+3 t^{9}+t^{8}+3 t^{6}+2 t^{5}+t^{4}+t^{3}+t+3\right)$.

The roots of the degree 20 factor of $\widetilde{\phi}(t)$ are not integral, and hence they are not roots of unity. We conclude that $\rho\left(\bar{S}_{3}\right) \leqslant 2$. By [vLui07], we have $\rho(\bar{S}) \leqslant \rho\left(\bar{S}_{3}\right)$, so $\rho(\bar{S}) \leqslant 2$. It follows that $S$ (and $\bar{S}$ ) has Picard rank 2. This concludes the proof of part (iv). Finally, the nontriviality of the Clifford invariant follows from Proposition 2 and Corollary 3. 


\section{Auel, Bernardara, Bolognesi and Várilly-Alvarado}

A satisfying feature of Theorem 9 is that we can write out a representative of the Clifford invariant of $X$ explicitly, as a quaternion algebra over the function field of the K3 surface $S$. We first prove a handy lemma, of independent interest for its arithmetic applications (see, for example, [HV13, HVV11]).

Lemma 10. Let $K$ be a field of characteristic $\neq 2$ and let $q$ be a nondegenerate quadratic form of rank 4 over $K$ with discriminant extension $L / K$. For $1 \leqslant r \leqslant 4$ let $m_{r}$ denote the determinant of the leading principal $r \times r$ minor of the symmetric Gram matrix of $q$. Then the class $\beta \in \operatorname{Br}(L)$ of the even Clifford algebra of $q$ is the quaternion algebra $\left(-m_{2},-m_{1} m_{3}\right)$.

Proof. On $n \times n$ matrices $M$ over $K$, symmetric gaussian elimination is the following operation:

$$
M=\left(\begin{array}{cc}
a & v^{t} \\
v & A
\end{array}\right) \mapsto\left(\begin{array}{cc}
a & 0 \\
0 & A-a^{-1} v v^{t}
\end{array}\right),
$$

where $a \in K^{\times}, v \in K^{n-1}$ is a column vector, and $A$ is an $(n-1) \times(n-1)$ matrix over $K$. Then $m_{1}=a$ and the element in the first row and column of $A-a^{-1} v v^{t}$ is precisely $m_{2} / m_{1}$. By induction, $M$ can be diagonalized, using symmetric gaussian elimination, to the matrix

$$
\operatorname{diag}\left(m_{1}, m_{2} / m_{1}, \ldots, m_{n} / m_{n-1}\right) .
$$

For $q$ of rank 4 with symmetric Gram matrix $M$, we have

$$
q=\left\langle m_{1}\right\rangle \otimes\left\langle 1, m_{2}, m_{1} m_{2} m_{3}, m_{1} m_{3} m_{4}\right\rangle,
$$

so that over $L=K\left(\sqrt{m_{4}}\right)$, we have $q \otimes_{K} L=\left\langle m_{1}\right\rangle \otimes\left\langle 1, m_{2}, m_{1} m_{3}, m_{1} m_{2} m_{3}\right\rangle$, which is similar to the norm form of the quaternion $L$-algebra with symbol $\left(-m_{2},-m_{1} m_{3}\right)$. Thus the even Clifford algebra of $q$ is Brauer equivalent to $\left(-m_{2},-m_{1} m_{3}\right)$ over $L$.

Proposition 11. The Clifford invariant of the fourfold $X$ of Theorem 9 is represented by the unramified quaternion algebra $(b, a c)$ over the function field of the $K 3$ surface $S$, where

$$
a=x-4 y-z, \quad b=x^{2}+14 x y-23 y^{2}-8 y z,
$$

and

$$
c=3 x^{3}+2 x^{2} y-4 x^{2} z+8 x y z+3 x z^{2}-16 y^{3}-11 y^{2} z-8 y z^{2}-z^{3} .
$$

Proof. Projecting with center the plane $P$, we obtain a quadratic form $\left(\mathscr{O}_{\mathbb{P}^{2}}^{3} \oplus \mathscr{O}_{\mathbb{P}^{2}}(-1), q, \mathscr{O}_{\mathbb{P}^{2}}(1)\right)$ of rank 4 over $\mathbb{P}^{2}$ associated with the quadric bundle $\pi: \widetilde{X} \rightarrow \mathbb{P}^{2}$; see [HVV11, $\S 4.2$ ] or $[$ Kuz10, $\S 4]$ for the computation of the Gram matrix. Since $S$ is regular, $\operatorname{Br}(S) \rightarrow \operatorname{Br}(k(S))$ is injective; see [AG60] or [Gro68, Cor. 1.10]. By the functoriality of the Clifford algebra, the generic fiber $\beta \otimes_{S} k(S) \in \operatorname{Br}(k(S))$ is represented by the even Clifford algebra of the generic fiber $q \otimes_{\mathbb{P}^{2}} k\left(\mathbb{P}^{2}\right)$. Thus we can perform our calculations in the function field $k(S)$. In the notation of Lemma 10, we have $m_{1}=2 a, m_{2}=-b$, and $m_{3}=-2 c$, and the formulas follow immediately.

Remark 12. Contrary to the situation in [HVV11], the transcendental Brauer class $\beta \in \operatorname{Br}(S)$ is constant when evaluated on $S(\mathbb{Q})$; this suggests that arithmetic invariants do not suffice to witness the nontriviality of $\beta$ in this case. Indeed, using elimination theory, we find that the odd primes $p$ of bad reduction of $S$ are $5,23,263,509,1117,6691,3342589,197362715625311$, and 4027093318108984867401313726363. For each odd prime $p$ of bad reduction, we compute that the singular locus of $\bar{S}_{p}$ consists of a single ordinary double point. Thus by [HV13, Prop. 4.1 and Lemma 4.2], the local invariant map associated with $\beta$ is constant on $S\left(\mathbb{Q}_{p}\right)$ for all odd primes $p$ of bad reduction. By an adaptation of [HV13, Lemma 4.4], the local invariant map is also constant for odd primes of good reduction. 


\section{Cubic fourfolds containing a Plane and a quintic del Pezzo surface}

At the real place, we prove that $S(\mathbb{R})$ is connected, hence the local invariant map is constant. To this end, recall that the set of real points of a smooth hypersurface of even degree in $\mathbb{P}^{2}(\mathbb{R})$ consists of a disjoint union of ovals (that is, topological circles, each of whose complement is homeomorphic to a union of a disk and a Möbius band, in the language of real algebraic geometry). In particular, $\mathbb{P}^{2}(\mathbb{R}) \backslash D(\mathbb{R})$ has a unique nonorientable connected component $R$. By graphing an affine chart of $D(\mathbb{R})$, we find that the point $(1: 0: 0)$ is contained in $R$. We compute that the map projecting from $(1: 0: 0)$ has four real critical values, hence $D(\mathbb{R})$ consists of two ovals. These ovals are not nested, as can be seen by inspecting the graph of $D(\mathbb{R})$ in an affine chart. The Gram matrix of the quadratic form, specialized at $(1: 0: 0)$, has positive determinant, hence by local constancy, the equation for $D$ is positive over the entire component $R$ and negative over the interiors of the two ovals (since $D$ is smooth). In particular, the map $f: S(\mathbb{R}) \rightarrow \mathbb{P}^{2}(\mathbb{R})$ has empty fibers over the interiors of the two ovals and nonempty fibers over $R \subset \mathbb{P}^{2}(\mathbb{R})$, where it restricts to a nonsplit unramified cover of degree 2, which must be the orientation double cover of $R$ since $S(\mathbb{R})$ is orientable (the Kähler form on $S$ defines an orientation). In particular, $S(\mathbb{R})$ is connected.

This shows that $\beta$ is constant on $S(\mathbb{Q})$. We believe that the local invariant map is also constant at the prime 2 , though this must be checked with a brute force computation.

\section{REFERENCES}

ABB13 A. Auel, M. Bernardara, and M. Bolognesi, Fibrations in complete intersections of quadrics, Clifford algebras, derived categories and rationality problems, J. Math. Pures Appl., to appear (2013), arXiv:1109.6938. http://dx.doi.org/10.1016/j.matpur.2013.11.009

AG60 M. Auslander and O. Goldman, The Brauer group of a commutative ring, Trans. Amer. Math. Soc. 97 (1960), 367-409.

AT12 N. Addington and R. Thomas, Hodge theory and derived categories of cubic fourfolds, Duke Math. J., to appear (2014), arXiv:1211.3758.

BB13 M. Bernardara, and M. Bolognesi, Derived categories and rationality of conic bundles, Compositio Math., 149 (2013), no. 11, 1789-1817. http://dx.doi.org/10.1112/S0010437X13007392

BCP93 W. Bosma, J. Cannon, and C. Playoust, The Magma algebra system. I. The user language, Computational Algebra and Number Theory (London, 1993), J. Symbolic Comput. 24 (1997), nos. 3-4, 235-265. http://dx.doi.org/10.1006/jsco.1996.0125

BD85 A. Beauville and R. Donagi, La variété des droites d'une hypersurface cubique de dimension 4 , C. R. Acad. Sci. Paris Sr. I Math. 301 (1985), no. 14, 703-706.

Bea77 A. Beauville, Variétés de Prym et jacobiennes intermédiaires, Ann. Sci. École Norm. Sup. (4) 10 (1977), no. 3, 309-391.

Bea00 A. Beauville, Determinantal hypersurfaces, Dedicated to William Fulton on the occasion of his 60th birthday, Michigan Math. J. 48 (2000), 39-64. http://dx.doi.org/10.1307/mmj/ 1030132707

CS07 A. Canonaco and P. Stellari, Twisted fourier-mukai functors, Adv. Math. 212 (2007), no. 2, 484-503. http://dx.doi.org/10.1016/j.aim.2006.10.010

EJ08 A.-S. Elsenhans and J. Jahnel, K3 surfaces of Picard rank one which are double covers of the projective plane, Higher-dimensional geometry over finite fields, NATO Sci. Peace Secur. Ser. D Inf. Commun. Secur. 16, IOS, Amsterdam, 2008, 63-77.

Fan43 G. Fano, Sulle forme cubiche dello spazio a cinque dimensioni contenenti rigate rationali di quarto ordine, Comment. Math. Helv. 15 (1943), 71-80.

Gro68 A. Grothendieck, Le groupe de Brauer. II. Théorie cohomologique, Dix Exposés sur la Cohomologie des Schémas, North-Holland, Amsterdam, 1968, 67-87. 


\section{Auel, Bernardara, Bolognesi and Várilly-Alvarado}

Has99 B. Hassett, Some rational cubic fourfolds, J. Algebraic Geom. 8 (1999), no. 1, 103-114.

Has00_, Special cubic fourfolds, Compositio Math. 120 (2000), no. 1, 1-23.

HS05 D. Huybrechts and P. Stellari, Equivalences of twisted K3 surfaces, Math. Ann. 332 (2005), no. 4, 901-936. http://dx.doi.org/10.1007/s00208-005-0662-2

HT01 B. Hassett and Y. Tschinkel, Rational curves on holomorphic symplectic fourfolds, Geom. Funct. Anal. 11 (2001), no. 6, 1201-1228.

HV13 B. Hassett and A. Várilly-Alvarado, Failure of the Hasse principle on general K3 surfaces, J. Inst. Math. Jussieu 12 (2013), no. 4, 853-877. http://dx.doi.org/10.1017/S1474748012000904

HVV11 B. Hassett, A. Várilly-Alvarado, and P. Várilly, Transcendental obstructions to weak approximation on general K3 surfaces, Adv. Math. 228 (2011), no. 3, 1377-1404. http://dx.doi.org/10. 1016/j.aim.2011.06.017

KPS86 M.-A. Knus, R. Parimala, and R. Sridharan, On rank 4 quadratic spaces with given Arf and Witt invariants, Math. Ann. 274 (1986), no. 2, 181-198. http://dx.doi.org/10.1007/BF01457069

Kuz06 A. Kuznetsov, Homological projective duality for Grassmannians of lines, arXiv:math/0610957 (2006).

Kuz07 _ Homological projective duality, Publ. Math. Inst. Hautes Études Sci. (2007), no. 105, 157-220. http://dx.doi.org/10.1007/s10240-007-0006-8

Kuz08 _ Derived categories of quadric fibrations and intersections of quadrics, Adv. Math. 218 (2008), no. 5, 1340-1369. http://dx.doi.org/10.1016/j.aim.2008.03.007

Kuz10 , Derived categories of cubic fourfolds, Cohomological and geometric approaches to rationality problems, Progr. Math., vol. 282, Birkhäuser Boston Inc., Boston, MA, 2010, 219-243. http://dx.doi.org/10.1007/978-0-8176-4934-0_9

Laz10 R. Laza, The moduli space of cubic fourfolds via the period map, Ann. of Math. 172 (2010), no. 1, 673-711. http://dx.doi.org/10.4007/annals.2010.172.673

Loo09 E. Looijenga, The period map for cubic fourfolds, Invent. Math. 177 (2009), 213-233. http: //dx.doi.org/10.1007/s00222-009-0178-6

vLui07 R. van Luijk, K3 surfaces with Picard number one and infinitely many rational points, Algebra Number Theory 1, (2007), no. 1, 1-15. http://dx.doi.org/10.2140/ant.2007.1.1

May11 E. Mayanskiy, Intersection lattices of cubic fourfolds, arXiv:1112.0806 (2011).

Mur77 J.P. Murre, On the Hodge conjecture for unirational fourfolds, Nederl. Akad. Wetensch. Proc. Ser. A 80 (Indag. Math. 39) (1977), no. 3, 230-232.

Nik79 V. Nikulin, Integer symmetric bilinear forms and some of their geometric applications, Math. USSR-Izv. 14 (1979), 103-167.

Sch85 W. Scharlau, Quadratic and Hermitian forms, Grundlehren der Mathematischen Wissenschaften 270, Springer-Verlag, Berlin, 1985. http://dx.doi.org/10.1007/978-3-642-69971-9

Sho84 V.V. Shokurov, Prym varieties: theory and applications, Math. USSR-Izv. 23 (1984), 83-147. http://dx.doi.org/10.1070/IM1984v023n01ABEH001459

Tre84 S.L. Tregub, Three constructions of rationality of a cubic fourfold, Vestnik Moskov. Univ. Ser. I Mat. Mekh. (1984), no. 3, 8-14. Translation in Moscow Univ. Math. Bull. 39 (1984), no. 3, 8-16.

Tre93 - Two remarks on four-dimensional cubics, Uspekhi Mat. Nauk 48 (1993), no. 2(290), 201-202. Translation in Russian Math. Surveys 48 (1993), no. 2, 206-208. http://dx.doi.org/ 10.1070/RM1993v048n02ABEH003729

Voi86 C. Voisin, Théorème de Torelli pour les cubiques de $\mathbb{P}^{5}$, Invent. Math. 86 (1986), no. 3, 577-601. http://dx.doi.org/10.1007/BF01389270

Voi07a , Hodge theory and complex algebraic geometry I, Translated from the French by Leila Schneps, Reprint of the 2002 English edition. Cambridge Studies in Advanced Mathematics 76, Cambridge University Press, Cambridge, 2007. http://dx.doi.org/10.1017/ CB09780511615344 
Cubic fourfolds Containing a Plane and a Quintic del Pezzo surface

Voi07b Some aspects of the Hodge conjecture, Jpn. J. Math. 2 (2007), no. 2, 261-296. http: //dx.doi.org/10.1007/s11537-007-0639-x

Zuc77 S. Zucker, The Hodge conjecture for cubic fourfolds, Compositio Math. 34 (1977), no. 2, 199-209.

Asher Auel asher.auel@yale.edu

Department of Mathematics, Yale University, 10 Hillhouse Avenue, New Haven, CT 06511, USA

Marcello Bernardara marcello.bernardara@math.univ-toulouse.fr

Institut de Mathématiques de Toulouse, Université Paul Sabatier, 118 route de Narbonne, 31062

Toulouse Cedex 9, France

Michele Bolognesi michele.bolognesi@univ-rennes1.fr

Institut de Recherche Mathématique de Rennes, Université de Rennes 1, 263 Avenue du Général Leclerc, CS 74205, 35042 Rennes Cedex, France

Anthony Várilly-Alvarado av15@rice.edu

Department of Mathematics, Rice University MS 136, 6100 South Main Street, Houston, TX 77005, USA 